\title{
Context-Adaptive User Interface in Ubiquitous Home Generated by Bayesian and Action Selection Networks
}

\author{
Han-Saem Park, In-Jee Song, and Sung-Bae Cho \\ Department of Computer Science, Yonsei University \\ 134 Shinchon-dong, Seudaemun-gu, Seoul 120-749, Korea \\ \{schunya, sammy\}asclab.yonsei.ac.kr, sbcho@cs.yonsei.ac.kr
}

\begin{abstract}
Recent home theater system requires for users to control various devices such as TV, audio equipment, DVD and video players, and set-top box simultaneously. To obtain the services that a user wants in this situation, user should know the functions and positions of the buttons in several remote controllers. Since it is usually difficult to manipulate them and the number of the devices that we can control increases, we get to confuse more as the ubiquitous home environment is realized. Moreover, there are a lot of mobile and stationary controller devices in ubiquitous computing environment, so that user interface should be adaptive in selecting the functions that user wants and in adjusting the features of UI to fit in a specific controller. To implement the user and controller adaptive interface, we model the ubiquitous home environment and use the modeled context and device information. We have used Bayesian network to get the degree of necessity in each situation. Action selection network uses predicted user situation and necessary devices, and it selects necessary functions in current situation. Selected functions are used to construct adaptive interface for each controller using presentation template. For experiments, we have implemented ubiquitous home environment and generated controller usage $\log$ in this environment. We have confirmed the BN predicted user requirements effectively as evaluating the inferred results of controller necessity based on generated scenario. Finally, comparing the adaptive home UI with the fixed one by 14 subjects, we confirm that the generated adaptive UI is more useful for general tasks than the fixed UI.
\end{abstract}

Keywords: Adaptive user interface, ubiquitous home, Bayesian network, action selection network.

\section{Introduction}

People use remote controllers belonging to the appliances when they want to control them. If they purchase more appliances, the number of controllers is getting bigger according to one of appliances. For example, users have to control several devices such as cable TV set-top-box, DVD player, television, audio equipment, video, and DivX players to use a recent home theater system. Controllers for those devices have different interfaces by their companies though they looked similar. Thus, it is not easy to get accustomed to all controller interfaces [1]. Though the controllers have various 
functions, only about a third is used practically. If ubiquitous home is generalized, most home devices like lights and boilers as well as home appliances would be controlled using remote controllers. Therefore, it is required to study a context adaptive user interfaces that provide users necessary functions among many of them.

In this situation, PUC (Personal Universal Controller), automatically generating user interfaces in PDA or smart phones, has been investigated recently. J. Nichols and B.A. Myers in CMU developed the system, which can generate an optimized controller interface for smart phone using hierarchical menu navigation system [2] and presented HUDDLE, which generates automatically task-based user interface [1]. Besides, they verified that automatically generated interface has better usability in terms of the time efficiency and consistency than general interfaces for device control using user study [3]. These studies generated and provided the useful PUC, but they cannot consider user's context.

We implemented the ubiquitous home environment and used the space and device information. In addition to the user and sensor input, system have used Bayesian network to infer the necessity of devices given context. Also, action selection network has used the necessity of devices as input and selected the function which is necessary in current context. Adaptive user interface consists of these selected function using presentation template. Modeling using Bayesian networks provides good performance as effectively incorporating the domain knowledge [4]. We also modeled the user interface with action selection network, so it can adapt to the user input constantly.

For experiment, we have made the log of the use of devices based on scenario and infer the necessity of devices with this log. After that, we evaluated the proposed method using GOMS model. 14 subjects were asked to perform 10 tasks with both conventional fixed home UI and proposed adaptive home UI. The results showed that subjects dealt with the general tasks more effectively using the proposed UI than using conventional one.

\section{Related Works}

\subsection{Bayesian Networks for Context-Awareness}

Context can have several meanings. Dey defined context as any information that can be used to characterize the situation of an entity such as a person, place, or object that is considered relevant to the interaction between a user and an application, including the user and the application themselves [4]. Generally, context influences the user's preference to a service, and it does to controlling devices in home. It is because the devices user want to control can change according to user's context.

Bayesian network, one of the models that are used for context-awareness, is a model to infer the context and provide reliable performance with uncertain and incomplete information [5]. It can be modeled using the data and can be designed using expert knowledge, and has been used to classify and infer the mobile context based on these strengths. Korpipaa et al. in VTT utilized naive Bayes classifier to learn and classify the user's context [6]. E. Horvitz et al. in MS Research proposed the system that infers what the user concentrated in a certain time point in an uncertain environment [7]. 


\subsection{Action Selection Network}

Action selection network was presented by P. Maes for robot agent control [8]. Figure 1 illustrates an example of representative action selection network. In this network, competition of actions is the basic characteristic, and it is shown as a change

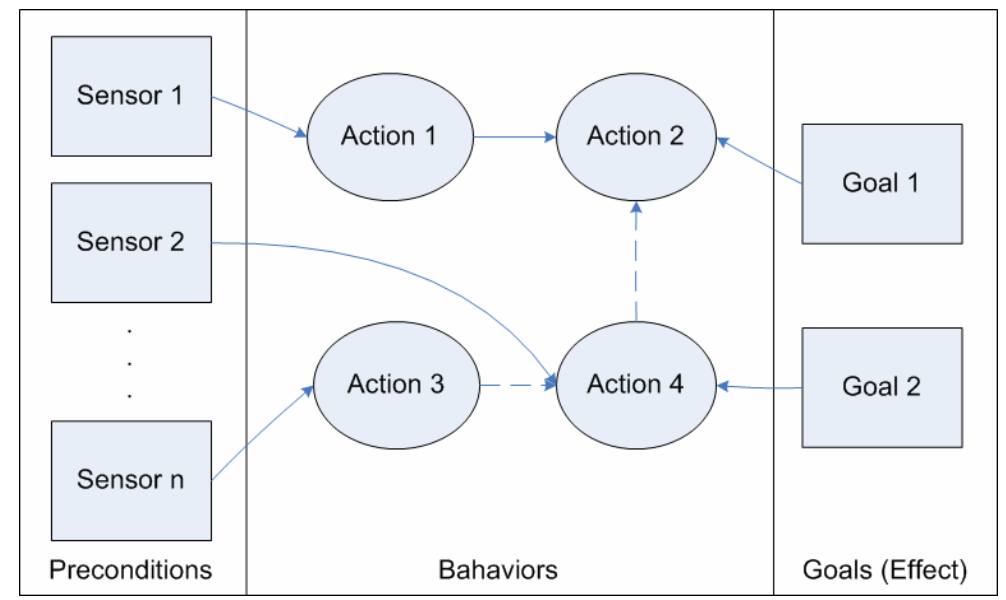

Fig. 1. An example of action selection network: Solid line represents predecessor link and dashed line represents successor link

of activation level in each action. An action with the highest activation level is selected after the activation spreading, it is calculated as follows. Nodes can have different types of links that encode various relationships and stimulate one another, and each node has five components of preconditions, add list, delete list, activation level, and the executable code. Links divides into internal and external, and internal link subdivides into predecessor link, successor link and conflict link. External link is connected to sensor and goals.

If the value of a certain sensor $S_{1}$ is true and $S_{1}$ is in the precondition list of an action node $A$, a link from $S_{1}$ to $A$ is activated. If goal $G_{1}$ has an activation larger than zero and $G_{1}$ is in the add list of $A$, a link from $G_{1}$ to $A$ is activated. The procedure to select an action node executed at each step is as follows:

1. Calculate the excitation from sensors and goals.

2. Spread excitation along the predecessor, successor and conflictor links, and normalize the activation level, so the average becomes equal to a constant $\pi$.

3. Check any executable nodes, select the one with the highest activation level, execute it, and finish. A node is executable if all preconditions are satisfied and its activation level is greater than threshold. If there is no executable node, reduce the threshold and repeat the process.

Links are set by a designer according to the given task. Using this, the system can select the proper action for its goal given a set of sensor states. 


\section{Context Adaptive User Interface for Ubiquitous Home}

Figure 2 summarizes the process of proposed adaptive UI generation for ubiquitous home devices. To begin with, Bayesian network infers the necessary devices in current context. With this result and the description of devices and controllers, action selection network is constructed in order to select the necessary functions for each device. Finally, user interface for a given controller is generated using UI template.

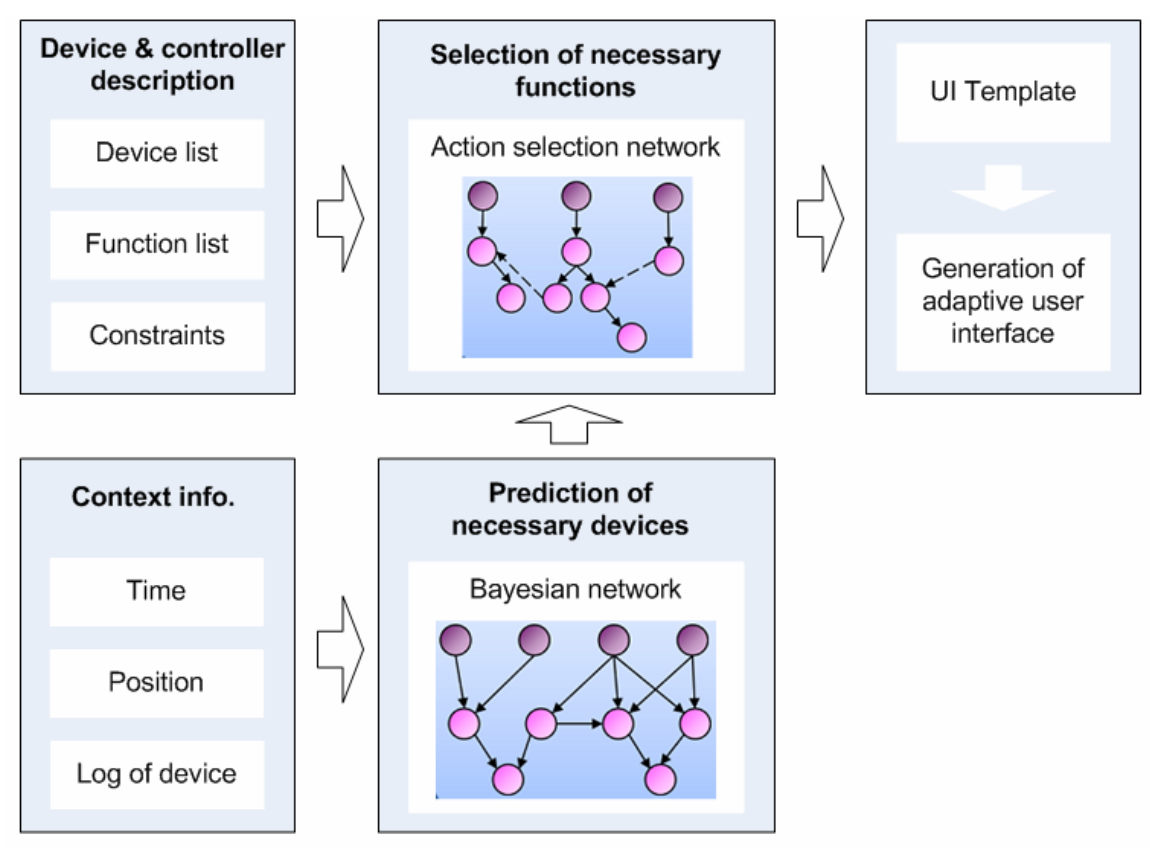

Fig. 2. Adaptive UI generation process

\subsection{Modeling Ubiquitous Home}

Ubiquitous home environment for adaptive user interface generation is modeled as follows.

- $\quad \mathrm{E}=\{$ Location_List, Device_List, Sensor_List $\}$

- $\quad$ Location_List $=\{$ Location1, Location2, ..., LocationN $\}$

- Device_List $=\{$ Device1, Device2, ..., DeviceN $\}$

- Sensor_List $=\{$ Sensor1, Sensor2, ..., SensorN $\}$

- Location $=\{$ Location_Name, Location_ID, Neighbors $\}$

- Device $=\{$ Device_Name, Device_ID, Location_ID, Function_List $\}$

- Function_List $=\{$ Function1, Function2, ..., FunctionN $\}$

- Function $=\{$ Function_Name, Function_ID, Function_Type, Function_Value, Add_List, Delete_List $\}$

- Sensor $=\{$ Sensor_Name, Sensor_ID, Sensor_Type, Sensor_Value $\}$ 
Ubiquitous home environment (E) includes location list, which tells locations of users, rooms and devices, device list including appliances like TV and video and equipments like boiler and lights, and sensor list, which observes the states of users and environments. Device information includes the name, location, and its functions, and function information includes functions of each device and the constraints. Function also includes Add_List and Delete_List for action selection network. Add_List has functions, which are desirable to be displayed with that function. Delete_List, on the other hand, has functions, which are not desirable to be displayed with. Location information is represented as a room name.

\subsection{Predicting Necessary Devices Using Bayesian Network}

We have used the Bayesian network to infer the devices that seem to be necessary for a given context in ubiquitous home. Since Bayesian network can be made by expert knowledge even though there are no or little data for learning [9], the system provides reliable performance in an uncertain home environment where it just set. After enough amount of $\log$ is obtained, it is possible to learn the Bayesian network. Personalized model can be learned using the log of individual users. We have used the K2 algorithm proposed by Cooper and Herskovits [10].

To calculate the necessity of each device, basic context such as user location, current time, and the date (whether the day is holiday or not) should be used as evidences. The logs of devices related to each device also have been used as evidences. Related devices mean the devices with similar function or in the same room. After all evidences are set, $\mathrm{BN}$ inference is conducted to predict the necessary devices.

\subsection{Selecting Necessary Functions Using Action Selection Network}

Once we get the necessities of each device, an action selection network is designed using Device_List (D) and Function_List (F) explained in section 3.1. Assuming these necessities of devices as a set of environmental nodes $E$ and the states of devices' functions as a set of functional nodes B, E and B are defined as Equation (1) and (2) using $\mathrm{F}$ and D.

$$
\begin{aligned}
& E=\left\{e_{i} \mid e_{i} \in D \wedge \operatorname{executable}\left(e_{i}\right)\right\} \\
& B=\left\{b_{i} \mid b_{i} \in F \wedge \operatorname{Executable}\left(b_{i}\right)\right\}
\end{aligned}
$$

After making the nodes, predecessor links, successor links, and conflictor links between these nodes are generated. Predecessor link is generated when Equation (3) is satisfied. Predecessor link, which connects two function nodes or a function node and an environment node, is generated when both nodes belong to the same device and they are related positively. Here, a positive relation occurs among functions that are likely to be executed after a certain function is executed. This link is used to make a hierarchical structure of functions in one device. Successor and conflictor links are used to represent the relations of functions in the different devices. Successor link is decided as Equation (4). It is similar to the predecessor link, but they are different in that successor link connects the functions of different devices and connects functional 
nodes only. Conflictor link is generated if Equation (5) is satisfied. Differing from the successor link, it is generated when two functions have negative relation (confliction).

$$
\begin{aligned}
& \operatorname{precondition}\left(n_{p}, n_{s}\right)=\left\{\begin{array}{c}
\text { if }\left\{\begin{array}{c}
\left(\left(n_{p} \subset B\right) \vee\left(n_{p} \subset E\right)\right) \\
\operatorname{Device}\left(n_{p}\right)=\operatorname{Device}\left(n_{s}\right) \\
\text { relation }\left(\operatorname{excute}\left(n_{p}\right), \operatorname{excute}\left(n_{s}\right)\right) \\
\text { otherwise }
\end{array}\right.
\end{array}\right. \\
& \operatorname{succesor}\left(n_{p}, n_{s}\right)=\left\{\begin{array} { c } 
{ 1 } \\
{ 1 }
\end{array} \text { if } \left\{\begin{array}{c}
\left(\left(n_{p} \subset B\right) \vee\left(n_{p} \subset E\right)\right) \\
\operatorname{Device}\left(n_{p}\right) \neq \operatorname{Device}\left(n_{s}\right) \\
\text { relation }\left(\operatorname{excute}\left(n_{p}\right), \operatorname{excute}\left(n_{s}\right)\right) \\
\text { otherwise }
\end{array}\right.\right. \\
& \operatorname{conflictor}\left(n_{p}, n_{s}\right)=\left\{\begin{array}{c}
\text { if }\left\{\begin{array}{c}
\left(\left(n_{p} \subset B\right) \vee\left(n_{p} \subset E\right)\right) \\
\operatorname{Device}\left(n_{p}\right)=\operatorname{Device}\left(n_{s}\right) \\
\operatorname{confliction}\left(\operatorname{excute}\left(n_{p}\right), \operatorname{excute}\left(n_{s}\right)\right) \\
\text { otherwise }
\end{array}\right.
\end{array}\right.
\end{aligned}
$$

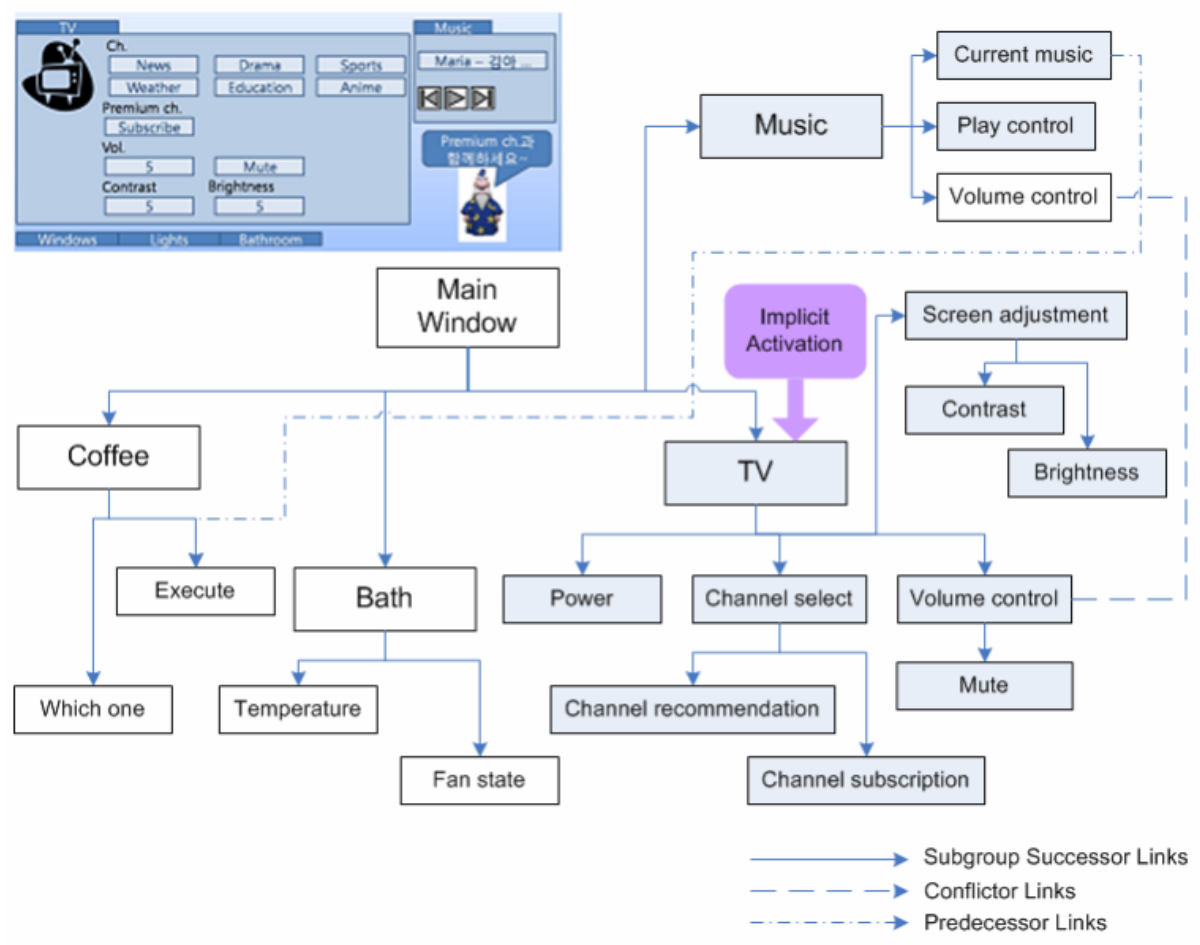

Fig. 3. Change of activation level in an action selection network and constructed user interface after the user controlled TV 
Constructed action selection network is basically represented as a tree, which has device nodes as parents and their functions as children, and each link is added to that tree based on the functional relations. In this network, activation functions are evaluated as $F: E \times B \rightarrow[0 \ldots 1]$. Using the inferred necessities of devices, a set of environment node $\mathrm{E}$ is made. After that, the procedure to select a necessary function in action selection network follows as explained in section 2.4. After the procedure, we can get an activation level of each function node. Active function node $b_{i}(t)$ is selected as Equation (6). We let the several functions be selected at once while the conventional action selection network does not, so user interface can use these selected functions to display at next step.

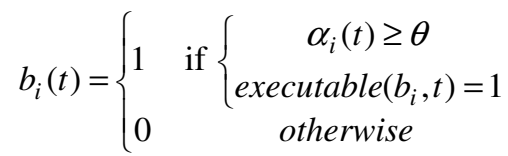

Constructed network is evaluated again when the device list user needs changes a lot or when user requests it. As explained in Figure 3, the necessity of TV gets larger if a user watches it, so network evaluation is updated. After evaluation, user interface includes more functions to control TV.

\section{Experiments}

For evaluation, we have conducted experiments in a simulated ubiquitous home environment. Comparing the proposed adaptive UI with the conventional fixed UI, we have confirmed that the adaptive UI provided better performance.

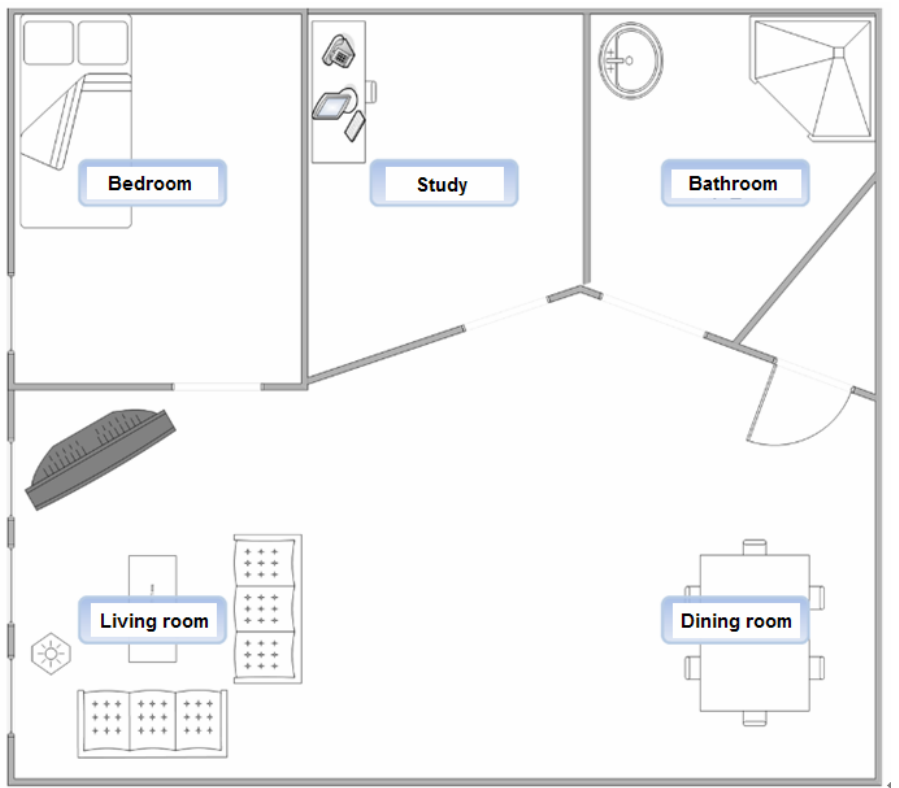

Fig. 4. A plane figure of ubiquitous home environment 


\subsection{Experimental Environment}

Simulated home environment is illustrated in Figure 4. It has 5 rooms of a living room, a dining room, a bedroom, a study, and a bathroom, and each room has devices summarized in Table 1. Lists for devices and functions are represented as XML.

Table 1. Devices and functions in each room

\begin{tabular}{|c|c|c|c|}
\hline \multirow{2}{*}{ Place } & \multirow{2}{*}{ Device } & \multicolumn{2}{|c|}{ Function } \\
\hline & & Name & Type \\
\hline \multirow{15}{*}{ Living room } & \multirow{5}{*}{ TV } & Power & Enum \\
\hline & & Channel & Enum \\
\hline & & Volume & Range \\
\hline & & Brightness & Range \\
\hline & & Light intensity & Range \\
\hline & \multirow{4}{*}{ Audio equipment } & Power & Enum \\
\hline & & Mode & Enum \\
\hline & & Play & Enum \\
\hline & & Volume & Range \\
\hline & \multirow{2}{*}{ Ceil light } & Power & Enum \\
\hline & & Light intensity & Range \\
\hline & \multirow{2}{*}{ Wall light } & Power & Enum \\
\hline & & Light intensity & Range \\
\hline & Window & Open/Close & Enum \\
\hline & Curtain & Open/Close & Enum \\
\hline \multirow{4}{*}{ Dining room } & \multirow{2}{*}{ Coffee maker } & Power & Enum \\
\hline & & Status & Enum \\
\hline & \multirow{2}{*}{ Ceil light } & Power & Enum \\
\hline & & Light intensity & Range \\
\hline \multirow{9}{*}{ Bedroom } & \multirow{2}{*}{ Ceil light } & Power & Enum \\
\hline & & Light intensity & Range \\
\hline & Window & Open/Close & Enum \\
\hline & Curtain & Open/Close & Enum \\
\hline & Bed & Fold/Unfold & Enum \\
\hline & \multirow{4}{*}{ Alarm } & Status & Enum \\
\hline & & Set (am/pm) & Enum \\
\hline & & Set (hour) & Range \\
\hline & & Set (minute) & Range \\
\hline \multirow{5}{*}{ Study } & \multirow{2}{*}{ Computer } & Power & Enum \\
\hline & & Status & Enum \\
\hline & Phone & Status & Enum \\
\hline & \multirow{2}{*}{ Ceil light } & Power & Enum \\
\hline & & Light intensity & Range \\
\hline \multirow{6}{*}{ Restroom } & \multirow{2}{*}{ Ceil light } & Power & Enum \\
\hline & & Light intensity & Range \\
\hline & \multirow{2}{*}{$\begin{array}{l}\text { Instantaneous } \\
\text { water heater }\end{array}$} & Power & Enum \\
\hline & & Temperature & Range \\
\hline & \multirow{2}{*}{ Fan } & Power & Enum \\
\hline & & Mode & Enum \\
\hline
\end{tabular}




\subsection{Usability Test for Adaptive User Interface}

To evaluate the usability of the proposed method, we assumed 10 situations that could happen in home environment and evaluated adaptive interface generated with GOMS model [11]. Situations are provided in Table 2.

Each situation has detailed tasks. For example, situation \#1 is "Having a breakfast while listening to music with audio" and its detailed tasks are as follows.

"Turn on the ceil light in a dining room $\rightarrow$ Turn on the coffee maker $\rightarrow$ Turn on the audio equipment in a living room $\rightarrow$ Set the frequency $\rightarrow$ Set the volume $\rightarrow$ Have a breakfast $\rightarrow$ Turn off the ceil light in a dining room"

Table 2. 10 situations for usability test

\begin{tabular}{cl}
\hline Number & \multicolumn{1}{c}{ Situation } \\
\hline 1 & Having a breakfast while listening to music with audio \\
2 & Watching TV in the evening \\
3 & Playing computer games \\
4 & Getting up from the bed in the morning \\
5 & Taking a shower \\
6 & Closing all windows and curtains \\
7 & Turning of all lights \\
8 & Watching TV while having dinner \\
9 & Having a phone conversation in a bed at night \\
10 & Going to sleep at night \\
\hline
\end{tabular}

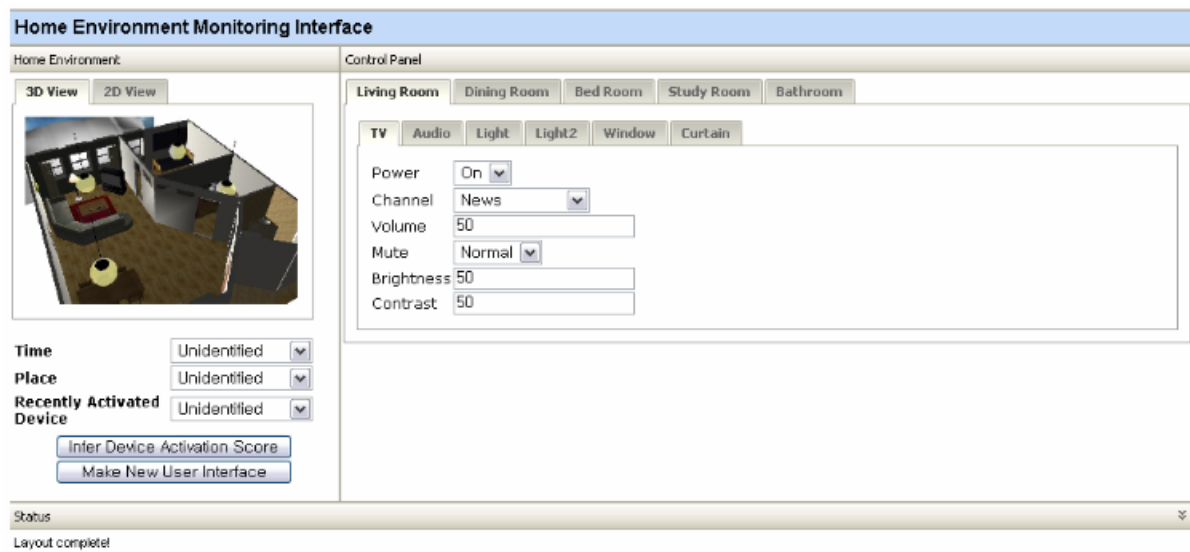

Fig. 5. A user interface used in current home network environment (A fixed UI only)

Using these situations, we have evaluated the user interfaces of Figure 5 and Figure 6 using GOMS. Figure 5 used tab-type interface for selecting place and device. To control a certain device, 2 steps of tab selection are required. This is an interface that 


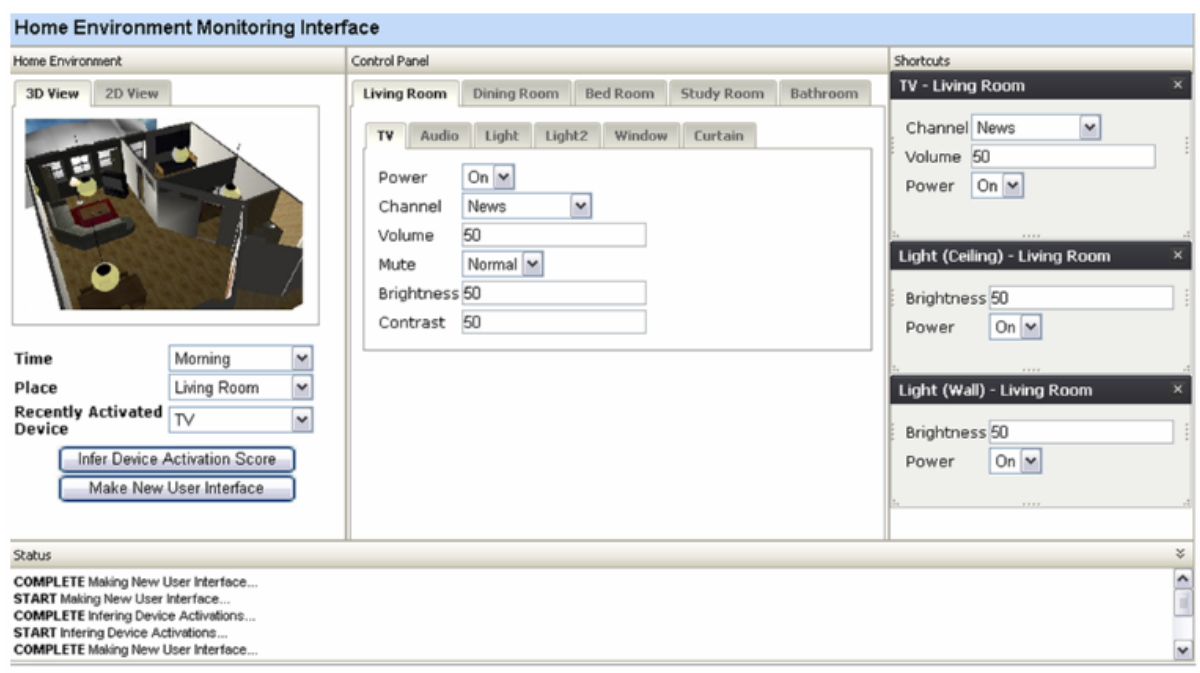

Fig. 6. A user interface including context-adaptive interface generated by proposed method (A fixed UI with an adaptive UI)

is based on a controller design of home network widely used. An interface in Figure 6 adds a context-adaptive interface at the right side. It changes according as the user controls the devices or the context changes. Using these two interfaces, we have compared the time that consumed for 14 users to perform the tasks in 10 situations. Figure 7 summarizes the result. When using an adaptive interface together, the reduction rate of time was $38.63 \%$ comparing to one only using a fixed user interface.

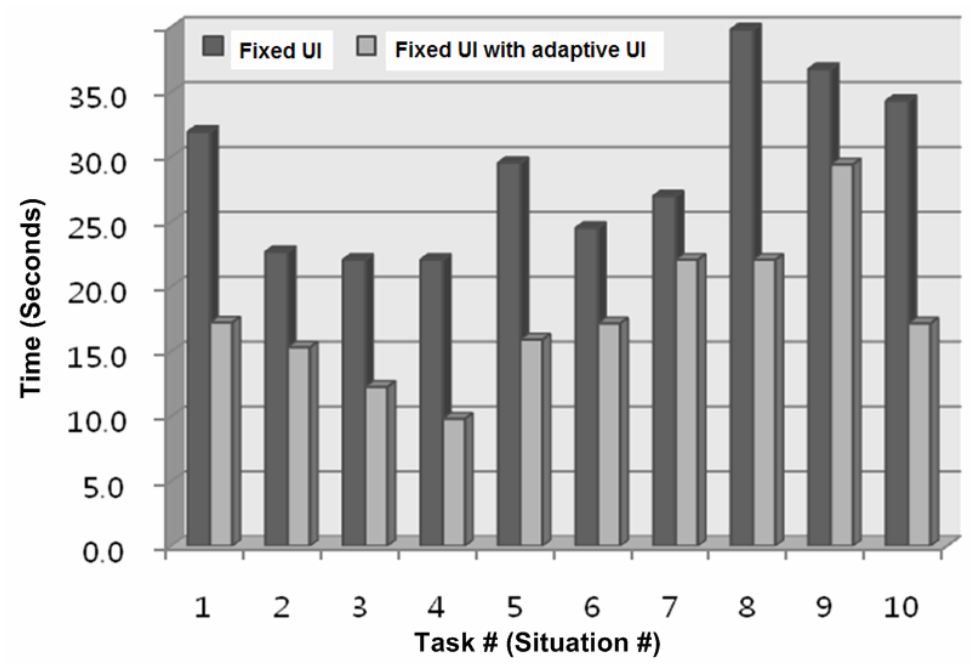

Fig. 7. Time consumed to perform tasks in 10 situations 


\section{Conclusion}

This paper proposed the method for context-adaptive user interface generation. There can be several devices and controllers in a ubiquitous home environment, proposed method generated the user interface considering both controller and devices. Bayesian network was used to infer the necessary devices, and an action selection network was used to select the necessary functions according as the context and user selection changes effectively. Finally, we have conducted the usability test of the user interface generated by the proposed method. For future work, we are planning to compare the proposed model with others.

\section{Acknowledgements}

This research was supported by the MKE (Ministry of Knowledge Economy), Korea, under the ITRC (Information Technology Research Center) support program supervised by the IITA (Institute of Information Technology Advancement) (IITA-2008(C1090-0801-0046))

\section{References}

1. Nichols, J., Rothrock, B., Chau, D.H., Myers, B.A.: Huddle: Automatically generating interfaces for systems of multiple connected appliances. In: UIST 2006, pp. 279-288 (2006)

2. Nichols, J., Myers, B.A.: Controlling home and office appliances with smart phones. IEEE Pervasive Computing 5(3), 60-70 (2006)

3. Nichols, J., Chau, D.H., Myers, B.A.: Demonstrating the viability of automatically generated user interfaces. In: CHI 2007, pp. 1283-1292 (2007)

4. Kleiter, G.D.: Propagating imprecise probabilities in Bayesian networks. Artificial Intelligence 88(1-2), 143-161 (1996)

5. Dey, A.K.: Understanding and using context. Personal and Ubiquitous Computing 5, 20 $24(2001)$

6. Korpipaa, P., Koskinen, M., Peltola, J., Makela, S.-M., Seppanen, T.: Bayesian approach to sensor-based context awareness. Personal and Ubiquitous Computing 7(2), 113-124 (2003)

7. Horvitz, E., Kadie, C.M., Paek, T., Hovel, D.: Models of attention in computing and communications: From principles to applications. Communications of the ACM 46(3), 52-59 (2003)

8. Maes, P.: How to do the right thing. Connection Science Journal 1(3), 291-323 (1989)

9. Cooper, G., Herskovits, E.A.: A Bayesian method for the induction of probabilistic networks from data. Machine Learning 9(4), 109-347 (1992)

10. Card, S., Moran, T.P., Newell, A.: The Psychology of Human Computer Interaction (1983) 